\title{
Cosmetic Outcome Following Cleft Lip and Palate Repair: A Case Series
}

\section{Abstract}

Cleft Lip and Palate are the most common congenital abnormalities of the orofacial structures. Along with the cosmetic problems, there may be associated feeding problems, speech problems and dental anomalies. Cleft Repair Surgeries are aimed at restoring the normal anatomy. The aesthetic and cosmetic outcome is a major determinant of the success of Cleft repair surgeries. We hereby present five pictorial cases of Cleft Lip and/or Palate who have undergone Repair surgeries at our institution, with their corresponding long-term cosmetic outcomes.

Keywords: Cleft lip; Cleft palate; Cleft repair surgery

Received: July 22, 2018; Accepted: December 13, 2018; Published: December 20, 2018

\section{Introduction}

Cleft Lip and Palate are the most common congenital abnormalities of the orofacial structures. With an incidence of 7 per 1000 children, this is one of the most common deformities among Indian children. Along with the cosmetic problems, there may be associated feeding problems, speech problems and dental anomalies. Cleft Repair Surgeries are aimed at restoring the normal anatomy. The displacement, deformation and underdevelopment of the muscles and facial skeleton are to be anatomically reconstructed near-normally, which encourages normal facial growth and function. Emphasis is placed on muscular reconstruction of the lip, nose and face, as well as muscles of the soft palate.

\section{Case Series}

Cleft Repair Surgeries have been performed by Dr. Y. N. Irkal, who is presently heading a renowned institution, Shreeya Hospital at Dharwad, Karnataka. The surgical outcomes are cosmetically satisfactory, as evident from the following photographs.

\section{Case 1}

A 6-month-old male child with bilateral cleft lip underwent surgical repair. On follow-up at the age of 5 years, upper lip has developed to be cosmetically aceptable (Figure 1).

\section{Case 2}

A 5-month-old male child with complaint of upper lip deformity, diagnosed to be bilateral cleft lip, underwent Cleft Repair Surgery.
Yallanasa NI and Amrut HB*

Department of General Surgery, Shreeya Multispeciality Hospital and Shreya College of Nursing, India

*Corresponding author: Amrut Hirulal Basava

”amrut1234@gmail.com

Department of General Surgery, Shreeya Multispeciality Hospital and Shreya College of Nursing, India

Citation: Yallanasa NI, Amrut HB (2018) Cosmetic Outcome Following Cleft Lip and Palate Repair: A Case Series. J Univer Surg. Vol.7 No.1:2 
common on the right side [3]. Most popular in CL/P surgery is the "RULE OF 10S" applied surgery which is consider best time to perform surgical intervention - infant is at least 10 weeks old, weighs 10 pounds, and has a haemoglobin level of $10 \mathrm{~g} /$ $\mathrm{dL}$, thereby surgeries are performed during the child's second to third month of life for health wellbeing among children [4].

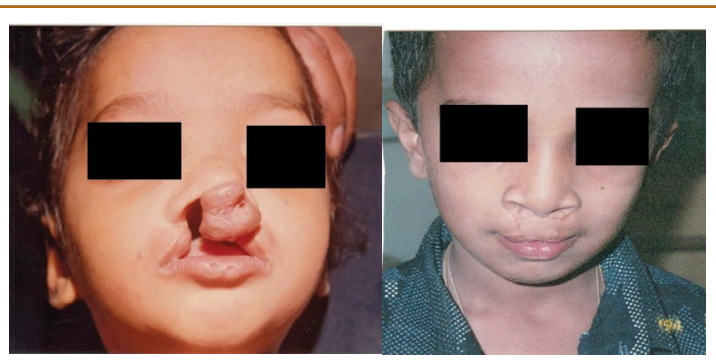

Figure 1 A male child (6 month old) with bilateral cleft lip underwent surgical repair, upper lip has developed to be cosmetically acceptable (at the age of 5 years).

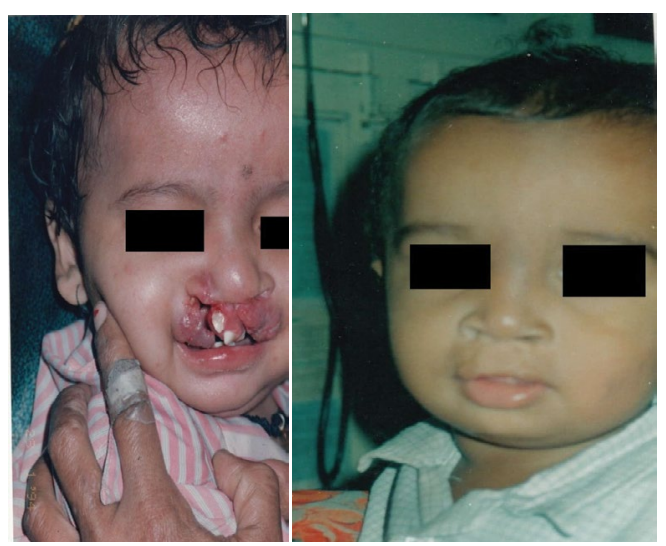

Figure 2 A male child (5 month old) with upper lip deformity, diagnosed to be bilateral cleft lip, underwent Cleft Repair Surgery. The cosmetic outcome at 4 years of age is satisfactory.

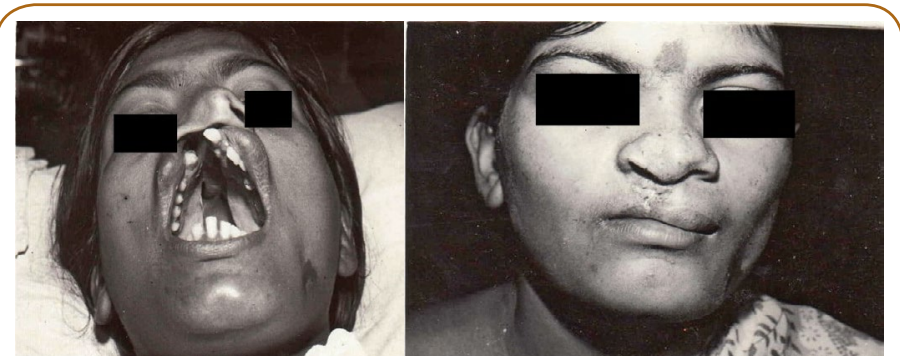

Figure 3 A 22 year old woman with unilateral Cleft lip and palate of orofacial deformity and speech problem underwent Cleft Repair Surgery, a good cosmetic outcome after1 year.

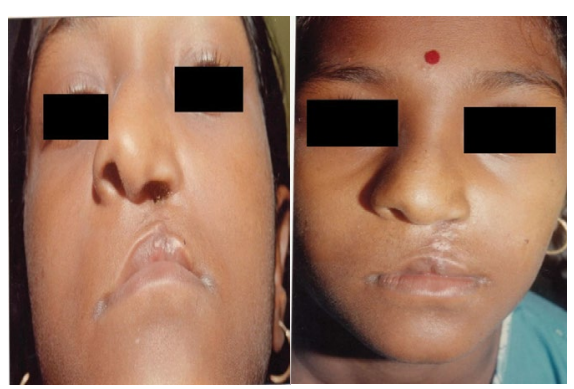

Figure 4 A 15 year old lady underwent surgery for unilateral cleft lip with residual deformity and asymmetrical upper lip, for improved symmetry and better cosmesis Underwent Revisional surgery (Z-plasty).

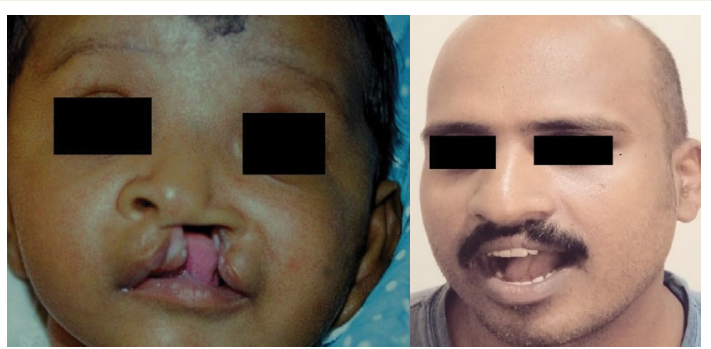

Figure 5 A male baby ( 3 month old) with unilateral cleft lip underwent Cleft Repair Surgery near-normal alignment of upper lip, and minimal scarring was seen at the age of 33 years.

Cleft Surgeries include Milliard technique and Palatoplasty. However, a Multidisciplinary approach is required for optimum management. Standardized photographs are currently the most commonly used medium for outcomes analysis worldwide. The Asher-McDade scale and The Unilateral Cleft Lip Surgical Outcomes Evaluation (UCL SOE) scale have been used to measure surgical outcome. The UCL SOE scores symmetry of 4 individual anthropomorphic components of the cleft repair (Cupid's bow, lateral lip, nose, and free vermillion) [5]. Photographs of cleft lip/palate shown to parents 'before and after' surgery are invaluable during parental counselling and support to allay fears and anxiety.

\section{Conclusion}

A satisfactory Cosmetic Outcome has been observed following Cleft Lip and Palate Repair Surgeries, as well as Revisional Surgeries at our institution. Surgical repair is indicated at an earliest possible age; the sooner it's performed, the better is the cosmetic outcome. The Photographs taken before and after Surgery portray the role of the Surgeon in improved aesthesis and facial development of Cleft patients. Better psychosocial lifestyle of the patient is noteworthy. 


\section{References}

1. Malathy I (2013) The Times of India, 1 in 1000 Indian kids born with a cleft lip. The Times Group, India.

2. Peter M, Julian L (2009) Addressing the challenges of cleft lip and palate research in India. Indian Journal of Plast Surg 42: S9-S18.

3. Uppal SK, Sheerin S, Rajinder KM, Ramneesh G, Ashok G, et al. (2016) Epidemiology and clinical profile of cleft lip and palate patients, in a tertiary institute in Punjab, India. J Cleft Lip Palate Craniofac Anomal 3: 32-35.

4. Tolorova M (1971) Orofacial clefts in Czechoslovakia: Incidence, genetics and prevention of cleft lip and palate over a 19-year period. Scand J Plast Reconstr Surg 21: 19-25.

5. Alex C, Carolina R, Gaurav D, Caroline T, Sarah MB, et al. (2017) Validation of a unilateral cleft lip surgical outcomes evaluation scale for surgeons and laypersons. Plast Reconstr Surg Glob Open 5: e1472. 